\title{
Response to Zucker Commentary on Gottschalk's (2003) 'Same-sex Sexuality and Childhood Gender Non-conformity: A Spurious Connection'
}

\author{
LORENE GOTTSCHALK
}

\begin{abstract}
In this reply to Zucker's commentary I point out that it is the meaning lesbians give to childhood gender non conformity rather than the frequency of reporting childhood gender non conformity that was discussed in my 2003 article. Indeed I did not, and do not, challenge the frequency of such reports. I also pointed to the importance of the historical, social and political context in which sexual identity formation occurs, and the influence of context on findings, and I reiterate this point in my response. Most of the articles cited by Zucker did not specifically explore the relationship between childhood gender non conformity and adult sexuality. One of the articles cited by Zucker actually supports my argument by commenting on the importance of context. I conclude that it might be heterosexual men who are the deviation from the norm and not gay men, lesbians and heterosexual women. I suggest that this is an important area for further research.
\end{abstract}

KEYWORDS: Gender, gender non conformity, childhood gender non conformity, homosexuality, same sex sexuality, lesbians, gay men

I argued in my article that the commonly believed relationship between childhood gender non-conforming behaviour and adult homosexuality is a spurious one. After reading Zucker's (2005) commentary with interest, I stand by my original assertion.

The frequency with which childhood gender non-conformity is reported by lesbians and gay men is well established and I did not, and do not, challenge the frequency of such reports. Bailey and Zucker's (1995) meta-analysis of all retrospective studies that have found that 'both homosexual men and women recalled more cross-typed behaviour during childhood than did their heterosexual counterparts' does not affect my argument in any way and thus was not cited in my paper.

Zucker's understanding of my article seems to be that I was challenging the frequency with which lesbians and gay men reported childhood gender non-conformity, when in fact my research revealed that it was the meaning that was given to childhood gender nonconformity by lesbians, that provides evidence that the connection is spurious. I also

Correspondence Address: Dr Lorene Gottschalk, Senior Lecturer, Management and Human Resource Management, School of Business, University of Ballarat, Ballarat, Victoria 3353, Australia; Email: 1.gottschalk@ballarat.edu.au

Journal of Gender Studies, Vol. 14, No. 2 July 2005, pp. 153-158

ISSN 0958-9236 Print/ISSN 1465-3869 $\quad$ c) 2005 Taylor \& Francis Group Ltd

http://www.tandf.co.uk/journal DOI: 10.1080/09589230500133643 
pointed out problems with existing research such as, the design of the study, methods of sampling, data collection and analysis used in many of the existing studies, including many of those cited by Zucker. For example the lack of study into childhood gender non-conformity and heterosexuality. Those few studies that do include both heterosexual women and men, and gay men and lesbians, raise questions rather than give answers. I used the studies of Phillips and Over (1995) and Bem (1996) to make this point.

Importantly most of the existing research is positivistic and thus misses two important factors. One is the meaning given to childhood gender non-conformity by the participants themselves. The second factor is the issue of reflexivity. That is, recognition by the researcher of the changing ideological context (the historical, social and political context) in which events occur. This includes recognition of the context of the participants and importantly of the researcher herself or himself. The personal context of the researcher can affect the design of the research, recruitment of the sample, the collection of the data and of course the interpretation of the data. When the researcher comes from a different historical, social and political context than their respondents (as they frequently do) the researcher is often not in a position to be able to anticipate the influence of these phenomena and thus include them in the design of their research instruments.

As I mentioned in my paper "many of the lesbians in my research did not speak of gender non-conformity in childhood' (Gottschalk, 2003a, p. 41) that is, they were gender conforming; however, many did. Indeed had I not followed up my research with qualitative in-depth interviews and specifically questioned lesbians who participated in my study about their understanding of, and the meaning they gave to, childhood gender nonconformity, my findings could well have been added to the body of literature that has found that many lesbians report gender non-conformity in childhood. It was my use of triangulation (qualitative as well as quantitative) methods of data collection that revealed the inconsistencies and contradictions in participants' understandings about gender nonconformity, how they experienced it and what meaning they gave to it.

Zucker questioned my methods of sampling and demographic data. I will start my response by briefly providing additional information about this. My participants were recruited through newspaper advertisements, fliers left at lesbian social clubs and venues, interviews on gay radio, networking and snowballing. The participants initially completed questionnaires which included a section for contact details if they agreed to be interviewed. All of the fifty-six women interviewed had completed questionnaires. The questionnaire included three questions relating to gender non-conformity each of which included provision for qualitative commentary. Thus at the interviews I was able to follow up and probe claims made in the questionnaires.

As can be seen even from the studies cited by Zucker, the sampling methods used in research involving homosexuals are frequently problematic. For example Loehlin and McFadden (2003) who studied otoacoustic emissions, auditory evoked potentials, and traits related to sex and sexual orientation state that most of their sample were college students, but some, especially in the homosexual and bisexual groups were from offcampus. This method of recruiting the sample may have influenced the findings. Strong et al. (2000, p. 436) acknowledged a sampling limitation in that many of the heterosexual men were recruited from masculine orientated social clubs such as fraternities, thus they may have been reluctant to admit to childhood gender non-conformity. The gay men were recruited from gay social clubs and thus may have been more open to admitting feminine behaviours. Bailey and Oberschneider (1997, p. 436) in their study of sexual orientation 
and professional dance could only find one lesbian dancer 'due to their apparent rarity' therefore they were not able to comment on lesbian dancers and childhood gender nonconformity. Yet most participants were recruited through personal networks and snowballing. That they could not find lesbian dancers through their personal network and snowballing does not necessarily mean that they are rare (though it may do). This is an example of how easily women's experiences can be excluded from a study and why so few studies have included lesbians.

The sampling for my study was as good as can be done for a cohort that is partially 'invisible'. No valid assessment is able to be made of the extent to which the women who completed and returned questionnaires, is representative of lesbians. A thorough demographic profile of lesbians (or homosexuals) in the state of Victoria (and I suspect elsewhere) does not exist, and indeed would be difficult to establish. Many women living in close loving relationships with a same-sex partner do not necessarily identify as a lesbian. There are also women (and men) who live in heterosexual marriages who identify as homosexuals but do not live as homosexuals, or alternatively identify as heterosexual even though they are in sexual relationships with a person of the same sex.

My study was an historical cohort analysis so the ages of my participants ranged from twenty years old to seventy-nine. They were divided into cohorts of women who identified as a lesbian prior to 1970, between 1970 and 1985, and since 1985. The latter group was divided into two groups, under and over thirty years of age. This is because of the sizeable number of women who identify as lesbians as adults. Each cohort category was significant enough to complete chi square statistics (see Gottschalk, 2003b). All of the participants self-identified as lesbians. My sample did not include heterosexual women.

One of the major flaws of most studies that make a connection between childhood gender non-conformity and adult homosexuality, is either the exclusion of heterosexual control groups or the total lack of comparison between lesbians and gay men and their heterosexual counterparts. I became aware of this lack as I was analysing interview data and noted the contradictory meanings given to childhood gender non-conformity. As I pointed out in my article in the discussion of the work of Phillips and Over (1995) and Bem (1996) it is also necessary to look at the overall frequency of childhood gender nonconforming behaviour as well as participant's interpretation of it. Their articles made it clear that heterosexual women are more gender non-conforming in childhood than gay men. This revelation resulted in a deeper analysis of the literature. Therefore in my article I questioned:

- the meaning given to the gender non-conformity by lesbians and gay men;

- how historical, social and political contexts can change those meanings;

- why it is that more heterosexual women than gay men report childhood gender non-conformity; and

- how it is then, that childhood gender non-conformity can be so strongly linked with adult homosexuality.

Indeed what is remarkable is not the high incidence of childhood gender nonconformity reported by lesbians, gay men and heterosexual women but the low reporting of childhood gender non-conformity by heterosexual men. This is the anomaly. Why is this so? I will return to this point later in my response.

Firstly I will briefly comment upon the studies cited by Zucker. Many of those studies do not specifically explore the relationship between adult homosexuality and childhood 
gender non-conformity. I believe this is problematic relative to citing them as evidence that there is a connection between adult homosexuality and childhood gender nonconformity. The respondents in those studies may well have been gender non-conforming in childhood but the connection is not examined. For example, Strong et al. (2000) discuss gender non-conformity in the context of a focus on body dissatisfaction. Bailey and Oberschneider (1997) examined the relationship between adult gay male dancers and childhood gender non-conformity. As mentioned earlier, Loehlin and McFadden (2003) studied otoacoustic emissions, auditory evoked potentials, and traits related to sex and sexual orientation. In Whitam et al. (1998) there was almost no discussion of childhood gender non-conformity. Further the authors compared Brazil, Peru, the Philippines and the United States. Such a comparison is not valid due to the different understandings of homosexuality in different cultures. For example, according to Almaguer (1991), from the perspective of Chicano culture it is often the passive 'penetrated' partner who is considered to be the homosexual and deviant, but not the active 'penetrating' partner. The political dimension of gender is a particularly important consideration here.

The many quantitative studies that report high levels of childhood gender nonconformity have not explored the various reasons for childhood gender non-conformity from the perspective of the participants, nor do they seem to have defined the concept for their participants. This interesting and important question was addressed in one of the studies cited by Zucker, that of Safir et al. (2003) who pointed out that definitions of tomboyism (female childhood gender non-conformity) are inconsistent and contradictory. In my own study, almost without exception the women spoke of playing sports, both body contact and non-body contact sports, when asked to describe what it was they were doing when they were gender non-conforming. In their questionnaires these women had linked their 'childhood gender non-conformity' to their sexuality. I reject the idea that playing sports is gender non-conforming for girls, other researchers may not. I would have thought that rather than gender non-conforming this is a healthy activity for girls.

Finally I wish to elaborate on an important point I made earlier, that of the historical, social and political context of the participants and the researchers and the dominant ideology about childhood gender non-conformity. Whisman (1996, p. 20) noted that the ideology surrounding homosexuality was developed through androcentric research, that did not take into consideration the social and political dimensions of gender. It must be understood that all human behaviour occurs in a historical, social and political context.

My study was an historical cohort analysis and so I could observe how perceptions differed according to the historical context in which lesbian identity development occurred. Rather than repeat those points I refer the reader to pp. 4041 of my article (Gottschalk, 2003a). It was the historical cohort analysis that revealed most clearly the influence of the dominant ideological context on the way that lesbians understand and experience their sexuality at the time lesbian identity development occurs. This includes the dominant ideological context (values, attitudes and beliefs) about gender, homosexuality and the assumed relationship between gender and homosexuality.

The importance of the social and political context on gender non-conformity was discussed by Safir et al. (2003). This study, cited by Zucker, actually supports my argument. They report that in Israel, childhood gender non-conformity is higher among heterosexual women than in other western countries as sports and rough and tumble play is 'more likely to be considered masculine in other western societies' than in Israel (p. 405). They found that very few adult lesbians regarded themselves as masculine and that they 
scored higher on the femininity score of the Personal Attributes Questionnaire (PAQ) than on the masculinity scale. Their original hypothesis, that lesbians will be more masculine and androgynous than heterosexual women, 'did not receive strong support' (p. 408). They explain this seemingly unexpected finding by considering the social and political context of Israel and the contradiction of Israeli culture which is at the same time both conservative and progressive. According to the authors it is also a culture that encourages what other cultures would call gender non-conformity in girls.

Safir et al. (2003) also make the point that discussions of the phenomena of sissie boys and tomboys (childhood gender non-conformity) need to consider the political and social context. Tomboys and sissy girls are not the counterparts of the same thing. They are two quite different categories in how they are seen by others as well as how they see themselves. As discussed in my article, a social context that values masculine more than feminine is likely to influence not only the extent to which women and men are likely to engage in childhood gender non-conforming behaviour but also the extent to which they report childhood gender non-conformity. It is likely that heterosexual men are less likely to report childhood gender non-conformity or at least give a different meaning to it. Troiden commented on this as early as 1979. Conversely it is also understandable in such a political context that heterosexual women are quite comfortable reporting gender nonconformity.

In my article I suggest that it is the dominant beliefs about gender and homosexuality which influence the adult homosexual's understanding of same-sex sexuality. I suggest that the idea of a connection between childhood gender non-conformity and adult homosexuality is a 'chicken and egg argument'. Does the gender non-conformity result in adult homosexuality or do stereotypical beliefs about adult homosexuality result in the high recollection of gender non-conformity? I argue that gender non-conformity is a social construction which is based on androcentric understandings of gender. More research is needed here.

The important questions remain. Who decides what is gender conforming, or nonconforming behaviour for girls and boys? If there is truly a connection between childhood gender non-conformity and adult homosexuality why is it that there are more heterosexual women than homosexual men who are gender non-conforming? The anomaly that I see in the research is the low reporting of childhood gender non-conformity by heterosexual men. I would argue then that the deviation is heterosexual men (not gay men and lesbians) and suspect that this would be a fruitful area of research.

\section{References}

Almaguer, Tomas (1991) Chicano men: a cartography of homosexual identity and behaviour, Differences: A Journal of Feminist Cultural Studies, 3 (Summer).

Bailey, J. Michael \& Oberschneider, Michael (1997) Sexual orientation and professional dance, Archives of Sexual Behavior, 26(4), pp. 433444.

Bailey, J. Michael \& Zucker, Kenneth J. (1995) Childhood sex typed behavior and sexual orientation: a conceptual analysis and quantitative review, Developmental Psychology, 31(1), pp. 4355.

Bem, Daryl (1996) Exotic becomes erotic: a developmental theory of sexual orientation, Psychological Review, 103(2), pp. 320335.

Gottschalk, Lorene (2003a) Same sex sexuality and childhood gender non conformity: a spurious connection, Journal of Gender Studies, 12(1), pp. 3550.

Gottschalk, Lorene (2003b) From gender inversion to choice and back: changing perceptions of the aetiology of sexuality over three historical periods, Women's Studies International Forum, 26(3), pp. 221233. 
Loehlin, John C. \& McFadden, Dennis (2003) Otoacoustic emissions, auditory evoked potentials, and traits related to sex and sexual orientation, Archives of Sexual Behavior, 32(2), April, pp. 115127.

Phillips, Gabriel \& Over, Ray (1995) Differences between heterosexual, bisexual and lesbian women in recalled childhood experiences, Archives of Sexual Behaviour, 24, pp. 120.

Safir, Marilyn, Rosenmann, Amir \& Kloner, Orly (2003) Tomboyism, sexual orientation, and adult gender roles among Israeli women, Sex Roles, 48(9/10), pp. 401410.

Strong, Scott M., Singh, Devendra \& Randall, Patrick (2000) Childhood gender non conformity and body dissatisfaction in gay and heterosexual men, Sex Roles, 43(7/8), pp. 427439.

Troiden, R. (1979) Becoming homosexual: a model of gay identity acquisition, Psychiatry, 42, pp. 362373.

Whisman, Vera (1996) Queer by Choice: Lesbians, Gay Men and the Politics of Identity (New York: Routledge).

Whitam, Frederick L., Daskalos, Christopher, Sobolewski, Curt G. \& Padilla, Peter (1998) The emergence of lesbian sexuality and identity cross culturally: Brazil, Peru, the Philippines, and the United States, Archives of Sexual Behavior, 27(1), pp. 3156

Zucker, Kenneth J. (2005) Commentary on Gottschalk's (2003) Same sex Sexulaity and Childhood Gender Non conformity: A Spurious Connection, Journal of Gender Studies, 14(1), pp. 5560. 
